\title{
Statistical modeling of hypertension cases in Navrongo, Ghana, West Africa
}

\author{
${ }^{1}$ Nasiru Suleman and ${ }^{2}$ Solomon Sarpong \\ ${ }^{1,2}$ Department of Statistics, Faculty of Mathematical Sciences, University for Development \\ Studies, P. O. Box 24 Navrongo, Ghana, West Africa \\ 1sulemannasiru@yahoo.com, \\ kwesarp@yahoo.com \\ ${ }^{1}$ Corresponding author's phone: +233246609024
}

\begin{abstract}
Concerns about the dangers of cardiovascular diseases have been a major problem all over the world. Hypertension which is one of the commonest cardiovascular diseases is gradually usurping the minds of public health workers globally. The disease is the most common cause of morbidity and mortality all over the world. In this study, we employed the Box-Jenkins approach to model and forecast admissions and outpatient hypertension cases in Navrongo. The analysis showed that ARMA $(1,1)$ and ARMA $(3,2)$ models were adequate for modeling and forecasting admission and outpatient cases respectively. Although, our forecast shows a decreasing pattern in admission and outpatient cases, this does not provide grounds to infer that the disease is not prevalent but rather the Ministry of Health and public health workers should organize educative programs for the indigenes to help minimize the prevalence of hypertension.
\end{abstract}

Key words: Hypertension, Navrongo, Box-Jenkins, Forecast, Ghana.

\section{INTRODUCTION}

Hypertension, also known as high blood pressure is one of the cardiovascular diseases that are gradually usurping the minds of public health workers globally.

An individual is hypertensive if he or she experiences a repeated elevated blood pressure exceeding 140 over $90 \mathrm{mmHg}$ (a systolic pressure above 140 with diastolic pressure above 90). The disease is the most common cause of morbidity and maternal mortality all over the world. It was estimated that more than a quarter of the world's population was hypertensive in the year 2000 , a number totaling nearly one billion and the number is expected to increase to 1.56 billion people world wide by the year 2025 (Kearney et al., 2005).

In Ghana, the prevalence of hypertension in urban Accra was estimated to be $28.3 \%$ (crude) and $27.3 \%$ (age-standardized) (Amoah, 2003). Also, a study on the changing patterns of hypertension in four rural communities in Ghana showed prevalence of $25.4 \%$. The study revealed that of those with hypertension only $32.3 \%$ had prior knowledge of their condition and less than half of these were on treatment (Addo et al., 2006). A survey conducted by Burket (2006) on blood pressure in the Volta Region of Ghana reported a frequency of $32.8 \%$ for hypertension with percentages of male and female been $30.7 \%$ and $39.4 \%$ respectively. Cappucio et al. (2004) in their study in the Ashanti region divulge a prevalence of hypertension of $28 \%$ for the Ashanti tribe in Ghana. According to the World Health Organization (WHO) African Regional Consultation meeting report on global strategy on diet, physical activity and health, the risk for non-communicable diseases appear to be gaining importance in Ghana, with prevalence of high blood pressure estimated at $30-40 \%$ although prevalence data for survey are generally inadequate (WHO, Harare, 2003).

Knowing the pattern of this detrimental disease could help public health workers and government to organize educative programs for citizens on causes of hypertension and it associated problems.

We therefore employed the Box-Jenkins approach to model and forecast hypertension cases in Navrongo, Ghana.

\section{MATERIALS AND METHODS}

The study area for this research is Navrongo. Navrongo is located in the Upper East Region of Ghana, West Africa. To achieve the stipulated objective, we collected data on admission and outpatient hypertension cases recorded on quarterly 
basis from 2000 to 2010 from the War Memorial Hospital located in Navrongo. The data were model using Autoregressive Integrated Moving Average (ARIMA) stochastic model popularized by BoxJenkins (1976). An ARIMA $(p, d, q)$ model is a combination of Autoregressive (AR) which shows that there is a relationship between present and past values, a random value and a Moving Average model which shows that the present value has something to do with the past residuals. The ARIMA process can be defined as:

$\emptyset(B)\left(\Delta^{d} y_{t}-\mu\right)=\theta(B) e_{t}$

where

$y_{t}=$ admission or outpatient cases.

$\mu=$ the mean of $\Delta^{d} y_{t}$,

$\emptyset(B)=1-\emptyset_{1} B-\ldots-\emptyset_{p} B^{p}$

$\theta(B)=1-\theta_{1} B-\ldots-\theta_{q} B^{q}$

$\emptyset_{i}=$ The $i^{\text {th }}$ autoregressive parameter

$\theta_{i}=$ The $i^{\text {th }}$ moving average parameter

$p, q$ and $d$ denote the autoregressive, moving average and differenced order parameter of the process respectively.

$\Delta$ and $B$ denote the difference and backward shift operators respectively.

The estimation of the model consists of three steps, namely: identification, estimation of parameters and diagnostic checking.

Identification step: involve the use of the techniques to determine the values of $p, q$ and $d$. The values are determined by using Autocorrelation function (ACF) and Partial Autocorrelation function (PACF). For any ARIMA $(p, d, q)$ process, the theoretical PACF has non-zero partial autocorrelations at lags $1,2, \ldots, p$ and has zero partial autocorrelations at all lags, while the theoretical ACF has non-zero autocorrelation at lags $1,2, \ldots, q$ and zero autocorrelations at all lags. The non-zero lags of the sample PACF and ACF are tentatively accepted as the $p$ and $q$ parameters. For a non stationary series the data is differenced to make the series stationary. The number of times the series is differenced determines the order of $d$. Thus, for a stationary data $d=0$ and ARIMA $(p, d, q)$ can be written as $\operatorname{ARMA}(p, q)$.
Estimation of parameters: the second step is the estimation of the model parameters for the tentative models that have been selected.

Diagnostic checking: the estimated model must be check to verify if it adequately represents the series. Diagnostic checks are performed on the residuals to see if they are randomly and normally distributed. Here, the Anderson-Darling test for normality was used. Also, the ACF and PACF plot of the residuals were examine to check if the residuals are white noise. The correlation matrix of the estimated parameters was tested to check if any of the parameters are correlated in order to eliminate any of the correlated ones. An overall check of the model adequacy was made using the Ljung-Box $\mathbf{Q}$ statistics. The test statistics is given by:

$Q_{m}=n(n+2) \sum_{k=1}^{n}(n-k)^{-1} r_{k}^{2} \approx \chi_{m-r}^{2}$

where

$r_{k}^{2}=$ the residuals autocorrelation at lag $k$

$n=$ the number of residuals

$m=$ the number of time lags included in the test.

When the $p$-value associated with the $\mathbf{Q}$ is large the model is considered adequate, else the whole estimation process has to start again in order to get the most adequate model. Here all the tests were performed at the $5 \%$ level of significance.

\section{RESULTS AND DISCUSSION}

The maximum and minimum number of patients admitted with hypertension problem was 57 and 7 respectively. For the outpatient hypertension cases, the maximum was 365 and the minimum was 17 . The average number of outpatient hypertension problem was 116.1 and that of those on admission was 21.84. The coefficient of variation for admission and outpatient cases were 40.27 and 71.48 respectively. This shows that the admission cases were more stable than the outpatient cases. The instability in the number of outpatient cases could be ascribed to the higher number of outpatient hypertension problems encountered over the period. The rapid decay in the ACF and PACF as depicted in Figures (I and II) shows that the data for the admission and outpatient cases were stationary. For the admission cases the ACF was zero after lag two $(q=2)$ and the PACF was zero after lag one $(p=1)$. Thus, our tentative 
model for the admission cases was ARMA $(1,2)$. To identify the tentative model for the outpatient hypertension cases, the ACF and the PACF were both zero after lag three $(p=3, q=3)$. Our tentative model for the outpatient cases is therefore $\operatorname{ARMA}(3,3)$.
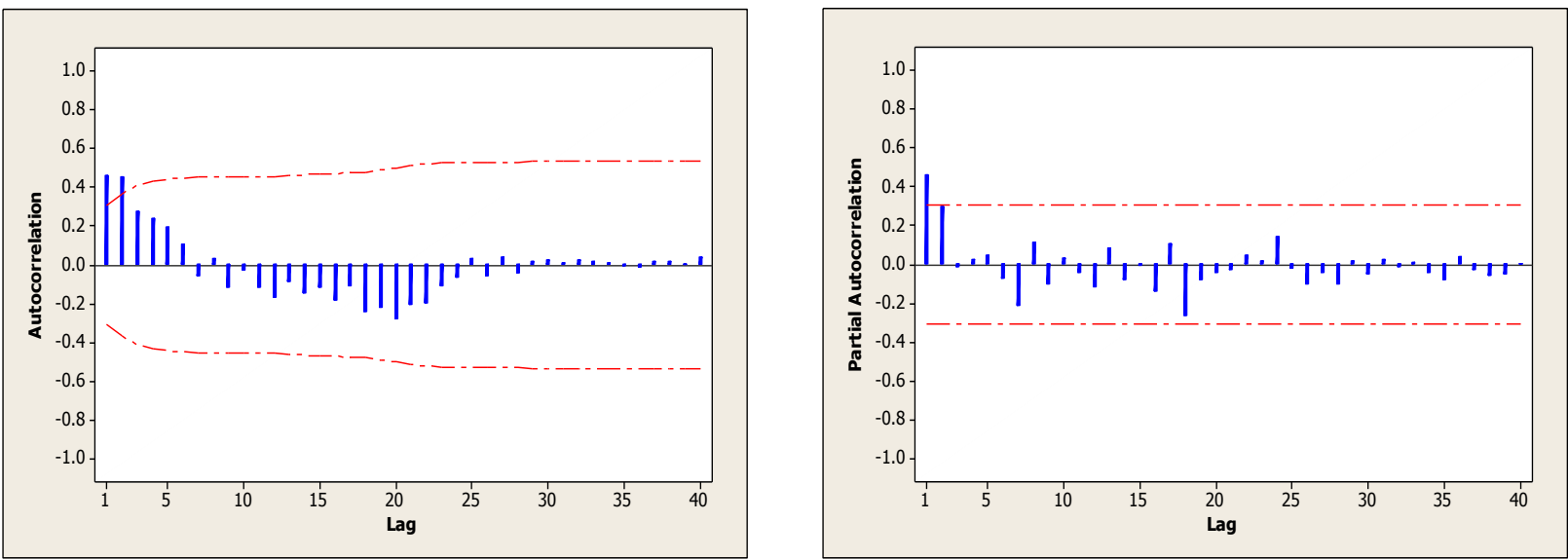

(a) ACF plot

(b) PACF plot

Fig 1: (a) ACF and (b) PACF plots for admission cases.
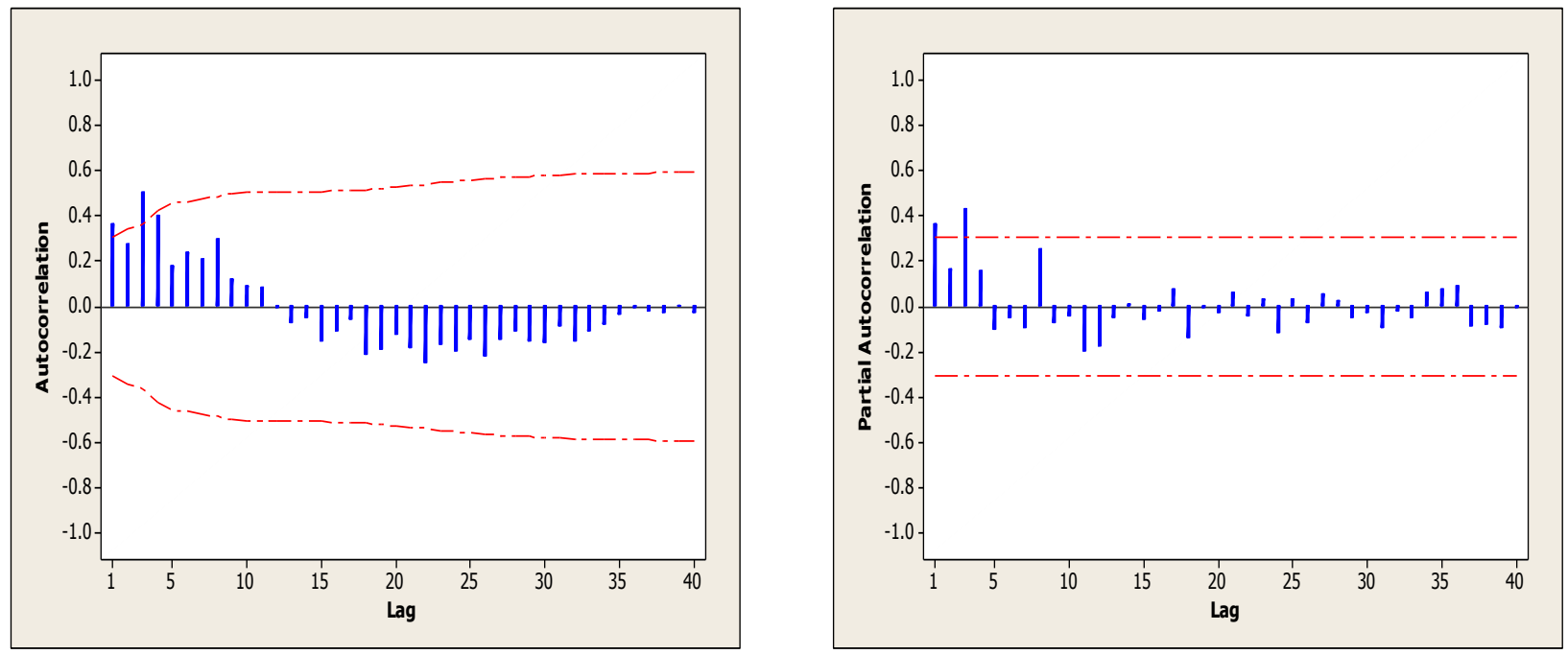

(a) ACF plot

(b) PACF plot

Fig 2: (a) ACF and (b) PACF plots for outpatient cases.

To ensure that these models are representative of our data we examined the ACF and PACF of the residuals resulted from fitting these models for our data. It was clear that no pattern was observed in the residuals for the ARMA models fitted. This shows that the models could be used to represent our data. After, we fitted ARMA $(1,2)$ and $\operatorname{ARMA}(3,3)$ for admission and outpatient hypertension cases respectively. After fitting the models, it was realized that for ARMA $(1,2)$ model, one of the Moving Average (MA) parameters was not significant so we dropped one of the MA parameters and estimated ARMA $(1,1)$. We examined the ACF and PACF of the residuals for this model and it was clear that the residuals are white noise. This implies that ARMA (1, 1 ) could be used to represent the admission cases. It 
is important to note that not only models with the minimum residual sum of squares is needed to fit the data but also, models with the least number of parameters are needed. Therefore the correlation matrix was tested to check if any of the parameters are strongly correlated in order to eliminate any of the correlated ones. Table I, shows the correlation between parameter values of the ARMA $(1,1)$ model for admission cases. There is no strong correlation between the parameter values, thus all the parameters are important to build the ARMA $(1,1)$ model. Therefore, ARMA $(1,1)$ was estimated for admission cases as depicted in Table II. The correlation matrix for ARMA $(3,3)$ model was examined. As shown in Table III, there was strong correlation between the model parameter values, thus some of the parameters need to be removed from the model. By eliminating any of the AR or MA parameters we will end up with the following models ARMA $(2,3)$ model with residual sum of squares of 171439 and ARMA $(3,2)$ model with residual sum of squares of 5255 . The result shows that $\operatorname{ARMA}(3,2)$ model is better than ARMA $(2,3)$ model. Thus ARMA $(3,2)$ was estimated for outpatient hypertension cases as shown in Table IV.
Also, an overall check of the models adequacy was made using the Ljung-Box statistics. Table VI, shows that the Ljung-Box statistics was large indicating the models were adequate.

Finally, we made a quarterly forecast of the admission and outpatient cases using ARMA $(1,1)$ and ARMA $(3,2)$ over a period of five years respectively. The forecast are shown in Table VII at the $95 \%$ confidence interval. The forecast for 2011 2012 admission cases for quarter one was 21.70 with a $95 \%$ confidence limit of $(6.76,36.64)$ and that of outpatient was 114.89 with a $95 \%$ confidence limit of $(-15.09,244.87)$. For $2015-2016$, the forecast for admission for quarter one was 21.57 with a $95 \%$ confidence limit of $(-7.81,50.95)$ and the outpatient cases were 114.63 with a $95 \%$ confidence limit of (186.27, 415.53). It must be noted that short term forecast is better as the errors of forecast increases with the increase of the periods of forecast.

Table 1: ARMA $(1,1)$ model correlation matrix

\begin{tabular}{|c|c|c|}
\hline parameter & $\mathrm{AR}(1)$ & $\mathrm{MA}(1)$ \\
\hline $\mathrm{AR}(1)$ & 1 & 0.243 \\
\hline $\mathrm{MA}(1)$ & 0.243 & 1 \\
\hline
\end{tabular}

Table 2: ARMA $(1,1)$ model parameter estimates

\begin{tabular}{|c|c|c|c|c|c|}
\hline TYPE & & COEFFICIENT & Standard error & $\mathrm{t}$-value & $p$-value \\
\hline $\mathrm{AR}(1)$ & & 0.9996 & 0.0225 & 44.47 & 0.000 \\
\hline $\mathrm{MA}(1)$ & & 0.5750 & 0.1302 & 4.42 & 0.000 \\
\hline
\end{tabular}

Table 3: ARMA $(3,3)$ model correlation matrix

\begin{tabular}{|c|c|c|c|c|c|c|}
\hline parameter & $\mathrm{AR}(1)$ & $\mathrm{AR}(2)$ & $\mathrm{AR}(3)$ & $\mathrm{MA}(1)$ & $\mathrm{MA}(2)$ & $\mathrm{MA}(3)$ \\
\hline $\mathrm{AR}(1)$ & 1 & -0.628 & -0.066 & 0.518 & -0.581 & 0.494 \\
\hline $\mathrm{AR}(2)$ & -0.628 & 1 & -0.628 & -0.081 & 0.291 & -0.268 \\
\hline $\mathrm{AR}(3)$ & -0.066 & -0.628 & 1 & -0.318 & 0.172 & -0.046 \\
\hline $\mathrm{MA}(1)$ & 0.518 & -0.081 & -0.318 & 1 & -0.762 & 0.837 \\
\hline $\mathrm{MA}(2)$ & -0.581 & 0.291 & 0.172 & -0.762 & 1 & -0.746 \\
\hline $\mathrm{MA}(3)$ & 0.494 & -0.268 & -0.046 & 0.837 & -0.746 & 1 \\
\hline
\end{tabular}

Table 4: ARMA $(3,2)$ model parameter estimates

\begin{tabular}{|c|c|c|c|c|c|}
\hline TYPE & COEFFICIENT & Standard error & $t$-value & $p$-value \\
\hline AR 1 & 0.9000 & 0.15805 & 5.70 & 0.000 \\
\hline AR(2) & -0.6351 & 0.1881 & -3.38 & 0.002 \\
\hline AR(3) & 0.6901 & 0.1481 & 4.66 & 0.000 \\
\hline MA(1) & 0.671 & 0.1294 & 5.18 & 0.000 \\
\hline MA(2) & -0.8141 & 0.1167 & -6.97 & 0.000 \\
\hline
\end{tabular}


In addition, the ACF and PACF of the residuals for ARMA $(1,1)$ and $\operatorname{ARMA}(3,2)$ were examined. As shown in Figures (III and IV) there is no pattern in the residuals. The residuals for these models were again tested for normality using the Anderson-Darling test. As shown in Table $\mathrm{V}$, the test shows that the residuals for these models were normally distributed.

Table 5: Anderson-Darling test statistics for Models

\begin{tabular}{|c|c|c|}
\hline Model & $\begin{array}{c}\text { Anderson- } \\
\text { Darling } \\
\text { statistics }\end{array}$ & $p$-value \\
\hline ARMA $(1,1)$ & 0.681 & 0.07 \\
\hline ARMA(3, 2) & 0.345 & 0.471 \\
\hline
\end{tabular}

Table 7: Forecast for admission and outpatient cases
Table 6: Ljung-Box statistics for Models

\begin{tabular}{|c|c|c|}
\hline Model & $\begin{array}{c}\text { Ljung-Box } \\
\text { statistics } \\
\text { (lag 36) }\end{array}$ & $p$-value \\
\hline ARMA $(1,1)$ & 19.7 & 0.976 \\
\hline ARMA(3, 2) & 16.6 & 0.983 \\
\hline
\end{tabular}

\begin{tabular}{|c|c|c|c|}
\hline Year & Quarter & Admissions cases & outpatient cases \\
\hline \multirow[t]{4}{*}{ 2011-2012 } & 1 & $21.70 \pm 14.94$ & $114.89 \pm 129.98$ \\
\hline & ॥ & $21.69 \pm 16.23$ & $159.44 \pm 133.35$ \\
\hline & III & $21.68 \pm 17.42$ & $215.46 \pm 142.43$ \\
\hline & IV & $21.67 \pm 18.54$ & $171.94 \pm 183.59$ \\
\hline \multirow[t]{4}{*}{$2012-2013$} & 1 & $21.66 \pm 19.60$ & $127.94 \pm 205.80$ \\
\hline & ॥ & $21.66 \pm 20.59$ & $154.64 \pm 210.60$ \\
\hline & III & $21.65 \pm 21.54$ & $176.58 \pm 219.28$ \\
\hline & IV & $21.64 \pm 22.45$ & $149.00 \pm 237.33$ \\
\hline \multirow[t]{4}{*}{ 2013-2014 } & 1 & $21.63 \pm 23.33$ & $128.67 \pm 248.53$ \\
\hline & II & $21.62 \pm 24.17$ & $143.04 \pm 253.68$ \\
\hline & III & $21.62 \pm 24.99$ & $149.84 \pm 261.05$ \\
\hline & IV & $21.61 \pm 25.77$ & $132.81 \pm 271.34$ \\
\hline \multirow[t]{4}{*}{ 2014-2015 } & 1 & $21.60 \pm 26.53$ & $123.08 \pm 278.47$ \\
\hline & II & $21.59 \pm 27.28$ & $129.83 \pm 283.26$ \\
\hline & III & $21.58 \pm 28.00$ & $130.33 \pm 289.13$ \\
\hline & IV & $21.58 \pm 28.70$ & $119.78 \pm 295.80$ \\
\hline \multirow[t]{4}{*}{ 2015-2016 } & 1 & $21.57 \pm 29.38$ & $114.63 \pm 300.9$ \\
\hline & II & $21.56 \pm 30.05$ & $117.03 \pm 305.03$ \\
\hline & III & $21.55 \pm 30.71$ & $115.20 \pm 309.61$ \\
\hline & IV & $21.54 \pm 31.35$ & $108.45 \pm 314.30$ \\
\hline
\end{tabular}


Am. J. Soc. Mgmt. Sci., 2011, 2(4): 377-383
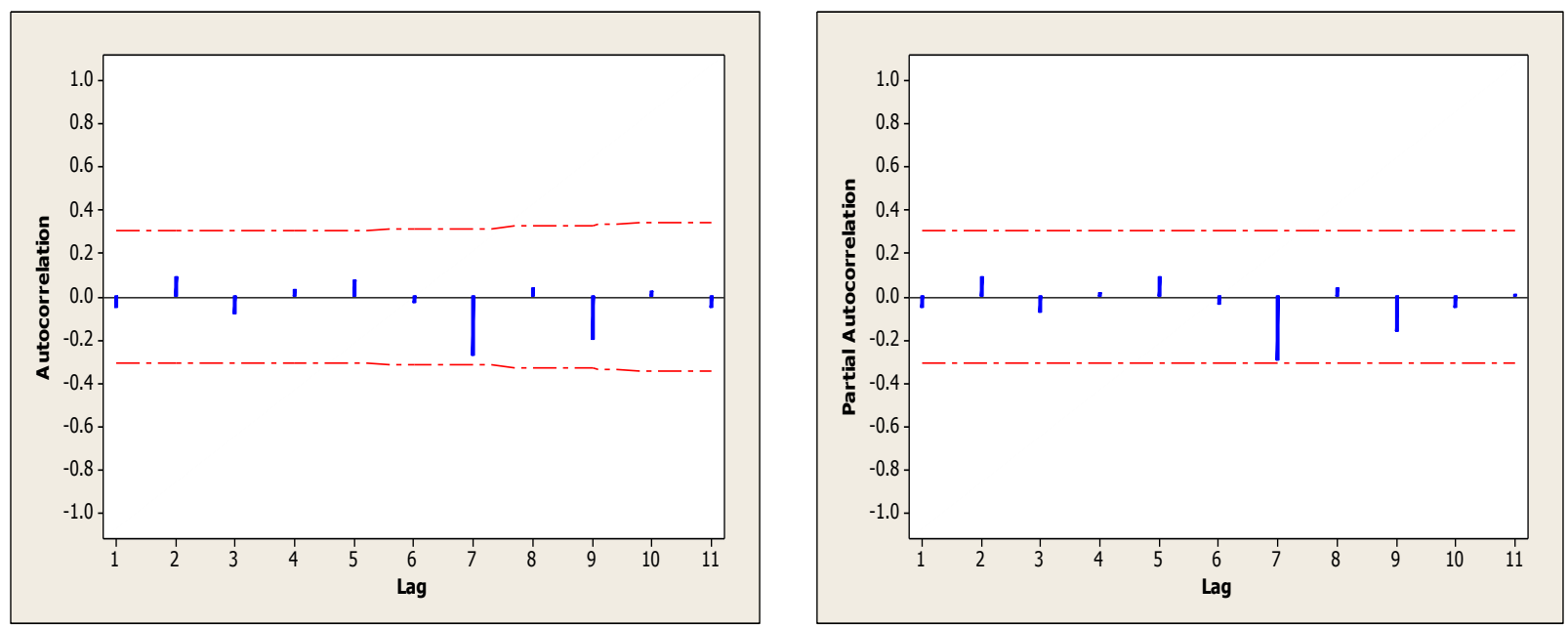

(a) ACF of ARMA $(1,1)$ residuals

(b) PACF of ARMA $(1,1)$ residuals

Figure III: Plots of (a) ACF and (b) PACF of ARMA $(1,1)$ model residuals.
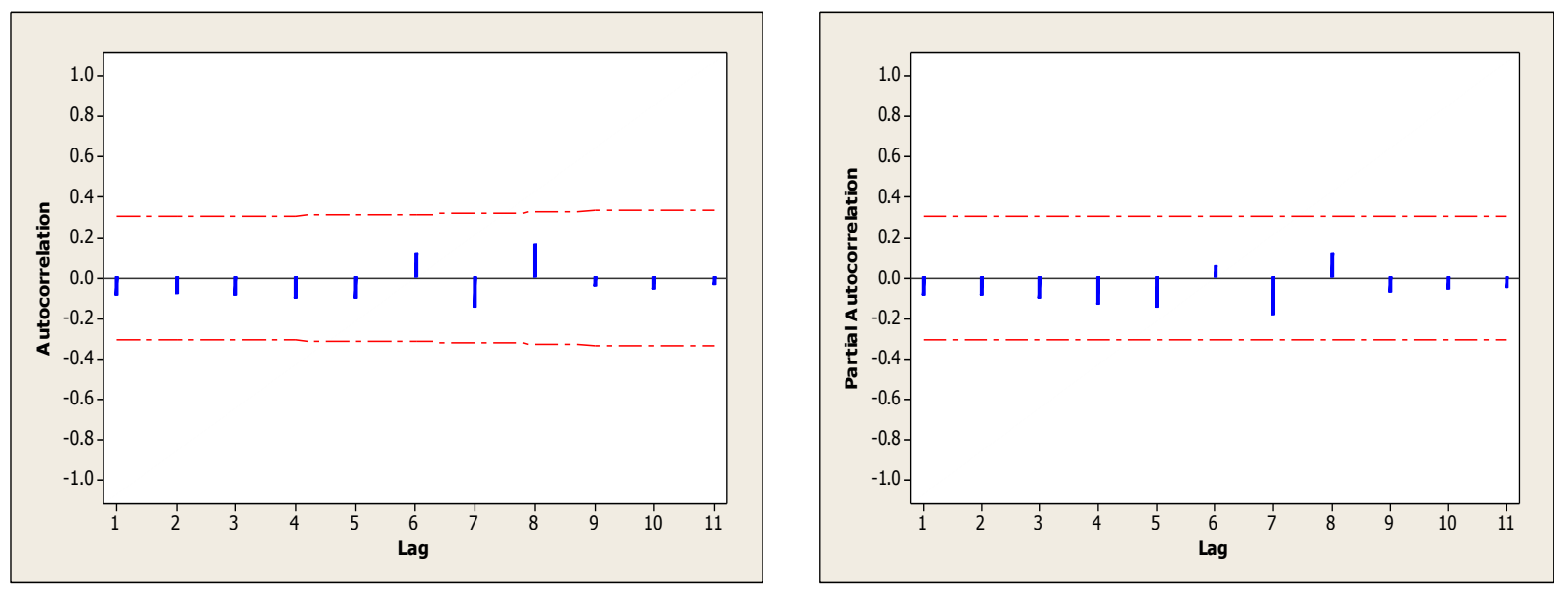

(a) $\operatorname{ACF}$ of $\operatorname{ARMA}(3,2)$ residuals

(b) PACF of ARMA $(3,2)$ residuals

Figure IV: Plots of (a) ACF and (a) PACF of $\operatorname{ARMA}(3,2)$ model residuals. 


\section{CONCLUSION}

Our forecast shows that there is a decreasing trend in the admission and outpatient hypertension cases in Navrongo. This decreasing pattern does not guarantee public health workers in the area to infer that the disease is not prevalent. Rather the Ministry of Health $(\mathrm{MoH})$ should liaise with health workers in the area to provide intensive education to the indigenes on risk factors of hypertension, causes, consequences and the need to report medical facilities with hypertension problem for treatment.

\section{REFERENCES}

Amoah A. G. B (2003). Hypertension in Ghana: A crosssectional community prevalence study in Greater Accra. Journal of Ethnicity and Diseases. 13: 310-315

Box G. E. P and G. M. Jenkins (1976). Time Series Analysis, Forecasting and Control. San Francisco, Holden-Day, California, USA.

Brent A. Burket (2006). Blood pressure survey in two communities in the Volta Region, Ghana, West Africa. Journal of Ethnicity and Diseases. 16: 292-294
Cappuccio F. P., Micah F. B., Emmett L., et al. (2004). Prevalence, detection, management and control of hypertension in Ashanti, West Africa. Journal of Hypertension. 43: 1017-1021 Juliet Addo, Albert G. B. Amoah, Kwadwo

A. Koram (2006). The changing patterns of hypertension in Ghana: A study of four rural communities in the GA District. Journal of Ethnicity and Diseases. 16: 894-898

Kearney P. M., Whelton M., Raynolds K., et al. (2005). Global burden of hypertension analysis of world data. Lancet. 365: 217-223

World Health Organization (2003). Global strategy on diet, physical activity and health: African Regional Consultation meeting report. Harare, Zimbabwe 2003. pp 12

Zuleat M. O (2007). The incidence of hypertension among adults in the Niger Delta Region of Nigeria. South East Asian Journal of Tropical Medicine and Public Health. 38(5):947-948 